\title{
Research on Job Shop Scheduling Method based on Genetic Algorithm under Uncertainty
}

\author{
Ya Gao, Yunfang Peng \\ School of Management \\ Shanghai University \\ Shanghai 200444, China \\ E-mail: gaoya302@sina.com
}

\begin{abstract}
The job shop scheduling problem with different due date windows is researched. A mathematical model is presented with the consideration of uncertain processing time which is presented by triangular fuzzy number. Furthermore, a genetic algorithm is developed to solve this problem. The algorithm generates the initial population by the method of integer coding, combining roulette method and elitist strategy is extended in the selection operation. Precedence operation crossover (POX) is used to ensure the feasibility of the offspring. In the end, an example is given to guarantee the validity of the model and algorithm.
\end{abstract}

Keywords- Job Shop Scheduling; Uncertainty; Earliness / Tardiness; Different Due Date Window; Genetic Algorithm

\section{INTRODUCTION}

Job Shop Scheduling Problem (JSSP) is a well-known scheduling problem, while the production scheduling optimization is the core of modern management techniques as well as advanced manufacturing technologies, which is a key factor to achieve efficient production of enterprise. With the continuous development of economic globalization, the customer needs is entering a new phase of diversification, the manufacturing industry is facing the problem of how to effectively organize many varieties of small batch production. JIT (Just in time) production method arises as a high-quality, low-cost mode of production under the condition of multi-species and small batch, it requires machining parts at the stipulated time, on-time delivery. Therefore, delivery of the goods is getting more attention as the evaluation index of scheduling problem, and the delivery window scheduling is more general expression of the scheduling problem in advance/tardiness, it rules that the completion time will not be punishment if it is between a certain range, otherwise it will be punished [1].

For previous researches on shop scheduling, less consideration is taken into about the uncertainty factors of the production, while the there are various uncertainties during the actual production process such as the uncertainty of processing time, uncertain product handling capacity and the emergency uncertainty [2]. The processing time is the core data of scheduling problem, Yang Hongan [3] proposed a new interval model based on selecting E/T penalty interval as optimization goal, using interval indicates the processing time. Lei [4] used a population-based neighborhood search (PNS) to optimize the interval makespan of the problem. For stochastic job shop scheduling problem, Azadeh [5] presented a heuristic algorithm based on computer simulation and artificial neural networks, and achieved optimal makespan by selecting the optimal scheduling rules. Hu [6] researched the fuzzy processing time and fuzzy due date of the job shop scheduling problem, and proposed a differential evaluation algorithm (DE) for multi-objective optimization. Li Junqing [7] proposed a hybrid algorithm (CROTS) which was combined with chemical reaction optimization and tabu search for fuzzy Job Shop scheduling. Yang Jianbin [8] extended the model to maximize customer satisfaction as the minimum scheduling objective under the fuzzy circumstances. The uncertainties described in above studies use the interval number, random variables and fuzzy mathematics method.

Job Shop Scheduling problem with different delivery window is studied, considering uncertainty of the product processing time, triangular fuzzy numbers is used to describe the uncertain processing time. Most previous research on fuzzy JSP problem, just set the target on processing time and delivery time, but in reality, policy makers are more concerned of the problems such as the production efficiency, cost, profit and others, therefore in this paper reducing the total cost is targeted, considering both the idle cost of the machine, and also the early and tardiness penalty value. A mathematical model is built with the consideration of uncertain processing time which is presented by triangular fuzzy number. An improved genetic algorithm is developed to solve this problem. Finally, a numerical example is given to show the convergence and effectiveness of the algorithm.

\section{DESCRIPTION OF THE PROBLEM}

\section{A. General Description of Scheduling problem}

Uncertain Job Shop Scheduling can be defined as: Suppose there are $\mathrm{m}$ machines, $\mathrm{n}$ jobs to be processed, each job $j$ composed by $d_{j}$ processes, in this paper $O_{i j}$ expresses the $i$ th operation on the job $j$. In each process the fuzzy processing time and delivery time are given in advance. The process needs to satisfy the following constraints:

(1) In the same time the same machine can only process one part;

(2) Each job can only be processed on a single machine at the same time, and each operation cannot be interrupted;

(3) The operations of different jobs do not have constraints;

(4) The different jobs have the same priority.

\section{B. Fuzzy numbers and its operations}

In this paper triangular fuzzy number is used to describe the uncertainty of the processing time, triangular 
fuzzy number is recorded as $\tilde{d}=\left(d^{L}, d^{M}, d^{U}\right)$. Its membership function is:

$$
\mathrm{u}(\mathrm{x})=\left\{\begin{array}{lr}
0 & x \leq d^{L} \\
\frac{x-d^{L}}{d^{M}-d^{L}} & d^{L}<x \leq d^{M} \\
\frac{d^{u}-x}{d^{U}-d^{M}} & d^{M}<x \leq d^{U} \\
0 & x>d^{U}
\end{array}\right.
$$$$
E_{\widetilde{\mathrm{q}}}=\frac{1}{2}\left(E_{1}+E_{2}\right)=\frac{a_{1}+2 a_{2}+a_{3}}{4}
$$

\section{MATHEMATICAL MODEL OF SCHEDULING PROBLEM}

The notations used in this study are listed as follows:

$n$ total number of jobs

$m$ total number of machines

Membership function is showed in Fig. 1:

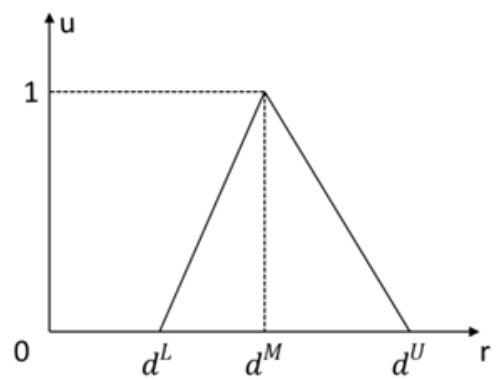

Figure 1. Membership function

Solving fuzzy shop scheduling problems, there are mainly three operations related to the triangular fuzzy number, including addition, the $V(\max )$ and sorting operations. Given two triangular fuzzy numbers: $\tilde{s}=$ $\left(s_{1}, s_{2}, s_{3}\right), \tilde{t}=\left(t_{1}, t_{2}, t_{3}\right)$.

(1) Addition operation. Addition operation is used to calculate the sum of the fuzzy start time and the processing time which is the completion time, just formula (1) as follows:

$$
\tilde{s}+\tilde{t}=\left(s_{1}+t_{1}, s_{2}+t_{2}, s_{3}+t_{3}\right)
$$

(2) $\mathrm{V}(\max )$ operation. $\mathrm{V}(\max )$ operation is used to compare the maximum of the release time and machine idle time, and then calculate the fuzzy start time of the job. The calculation formula (2) as follows:

$$
\tilde{s} \bigvee \tilde{t}=\left(s_{1} \bigvee t_{1}, s_{2} \bigvee t_{2}, s_{3} \bigvee t_{3}\right)
$$

(3) Evaluation of fuzzy numbers. Guidelines which is proposed by Sakawa [9]is conducted in this paper.

(4) The defuzzification of fuzzy number

For the ambiguity resolution the expected demand method [10] is available. Such as triangular fuzzy number $\tilde{q}=\left(a_{1}, a_{2}, a_{3}\right)$, so $E_{1}, E_{2}$ represent the bounds of Expect interval, respectively, then:

$$
\begin{aligned}
& E_{1}=a_{2}-\int_{a_{1}}^{a_{2}} f(x) d x \\
& E_{2}=a_{2}+\int_{a_{2}}^{a_{3}} g(x) d x
\end{aligned}
$$

Where:

So the expectation is:

$$
\begin{aligned}
& \mathrm{f}(\mathrm{x})=\frac{x-a_{1}}{a_{2}-a_{1}} \\
& \mathrm{~g}(\mathrm{x})=\frac{a_{3}-x}{a_{3}-a_{2}}
\end{aligned}
$$




$$
\begin{aligned}
& \tilde{c}_{j h} \leq \tilde{s}_{j(h+1)}+L\left(1-y_{k l j(h+1)}\right) \\
& s_{j h} \geq 0, \quad c_{j h} \geq 0
\end{aligned}
$$

Constraint (5) defines the completion time of each production. Constraint (6) ensures the precedence constrains based on each production. Constraint (7) represents the job completion time constraint. Constraints (8) (9) required that two operations cannot be processed simultaneously. Constraint (10) each parameter variables must be positive.

\section{SCHEDULING ALGORITHMS BASED ON GENETIC ALGORITHMS}

\section{A. Population initialization}

Population initialization gives an initial solution by way of chro mosome encoding. Using genetic algorithms to optimize production scheduling problem, it will be complicated using binary encoding or floating point encoding. It is not good for programming and computing, so integer coding method is used here, each chromosome represents all the job processing sequences. Randomly generated initial population according to the jobs and processing sequence, and these chromosomes is used to iterate as the initial point.

\section{B. Selection}

Select operation is a process to form a new population which is selected a strong vitality individual from an older population. This article researchers takes the roulette wheel selection and elitist approach to select operation. The greater the fitness is, the higher probability the individual is selected.

\section{Crossover}

Crossover recombines certain genes randomly in two parents' chromosomes to make two offspring, in order to generate a better solution. The aim is to combine good genes, so that the offspring can inherit better parents' genes. This paper is based on the POX (precedence operation crossover) operator, it is good to inherit parents' excellent characteristics and all the children are feasible [11].

For example, chromosome $\mathrm{C} 1=[1,3,1,2,4,4,3,2,1,3$, $2,4], \quad \mathrm{C} 2=[4,1,3,4,2,3,1,3,4,2,1,2]$, divided the $\mathrm{Job}$ set $\{1,2,3,4\}$ randomly into $\mathrm{J} 1=\{1,4\}$ and $\mathrm{J} 2=\{2,3\}$. The offspring chromosome after crossing were $\mathrm{C} 3=$ $[1,3,1,2,4,4,3,3,1,2,2,4], \quad \mathrm{C} 4=[4,1,3,4,2,3,1,2,4,3,1,2]$. The crossover process is as shown in Fig. 2. In the chromosomes crossover phase, whether each chromosome can be crossed is determined by crossover probability Pc.

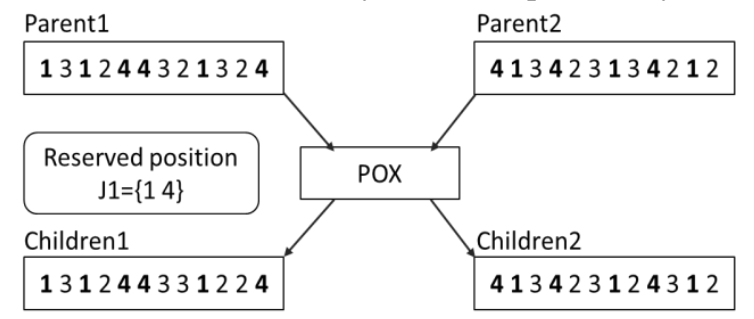

Figure 2. POX crossover operation

\section{Mutation}

Mutation operation aimed at improving the ability of local search algorithm, which helps maintain the diversity of evolution population, prevent premature local optimu m. In this paper, exchange mutation method is used for this operation. Firstly the mutation operator randomly selected individuals from the population. Then the two mutation positions are randomly selected and swapped. The process of mutation is as shown in Fig. 3.

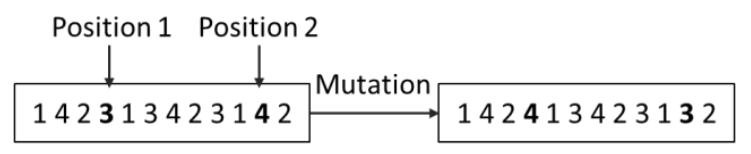

Figure 3. REM mutation operation

\section{NUMERICAL EXAMPLE}

In order to verify the efficiency of proposed genetic algorithm for solving fuzzy job shop scheduling, researchers use the same parameters in literature [12], $6 \times$ 6 and $10 \times 10$ examples were solved in this paper. The idle costs per unit of time, tard iness and early penalty values were 5,4 and 3 .

The algorithm was implemented in MATLAB and the tests were run on a computer with a $2.1 \mathrm{GHz}$ Intel CPU on the Windows 7 operating system. Job due date window is shown below in Table 1, 2 .

TABLE I. DUE DATE WINDOW OF 6×6 NUMERICAL EXAMPLE

\begin{tabular}{ccccccc}
\hline Jobs & 1 & 2 & 3 & 4 & 5 & 6 \\
\hline Due date & {$[30$,} & {$[35$,} & {$[20$,} & {$[32$,} & {$[30$,} & {$[40$,} \\
window & $40]$ & $40]$ & $28]$ & $40]$ & $35]$ & $45]$ \\
\hline
\end{tabular}

TABLE II.DUE DATE WINDOW OF 10×10 NUMERICAL EXAMPLE

\begin{tabular}{cccccc}
\hline Jobs & 1 & 2 & 3 & 4 & 5 \\
\hline Due date & {$[45$,} & {$[50$,} & {$[50$,} & {$[50$,} & {$[50$,} \\
window & $60]$ & $60]$ & $65]$ & $65]$ & $65]$ \\
\hline Jobs & 6 & 7 & 8 & 9 & 10 \\
\hline Due date & {$[45$,} & {$[45$,} & {$[50$,} & {$[45$,} & {$[50$,} \\
window & $60]$ & $60]$ & $60]$ & $60]$ & $60]$ \\
\hline
\end{tabular}

With the above method, taking the number of population Popsize $=40$, evolution generation Gennum $=$ 200, crossover probability $\mathrm{Pc}=0.8$, mutation probability $\mathrm{Pm}=0.6,20$ times simulation of the problem is conducted and taking the best result.

For the $6 \times 6$ problem, the minimum cost to get the optimal schedule is 518.75. Maximum fuzzy completion time is $(32,39,46)$. The minimu m cost convergence graph of $6 \times 6$ is shown in Fig. 4 . 


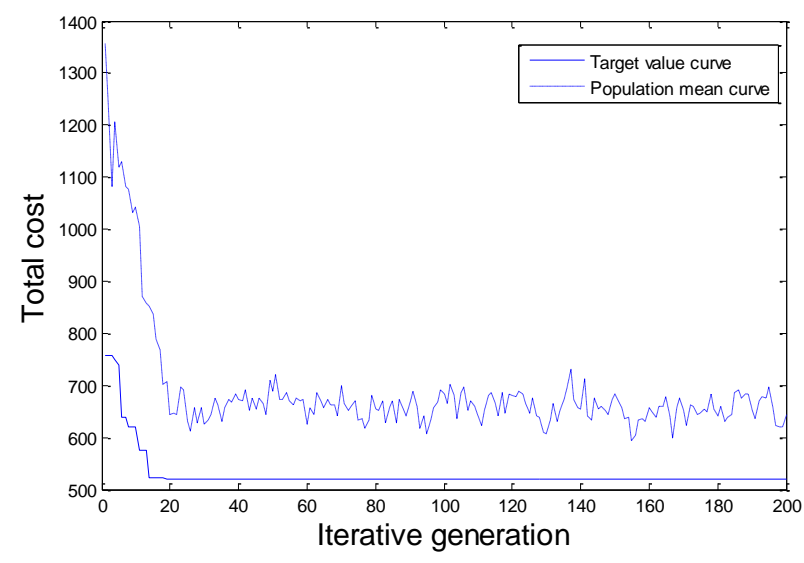

Figure 4. The minimum cost convergence graph of $6 \times 6$

For the $10 \times 10$ problem, the minimum cost to get the optimal schedule is 926.25. Maximum fuzzy completion time is $(28,49,63)$. The minimu m cost convergence graph of $10 \times 10$ is shown in Fig. 5 .

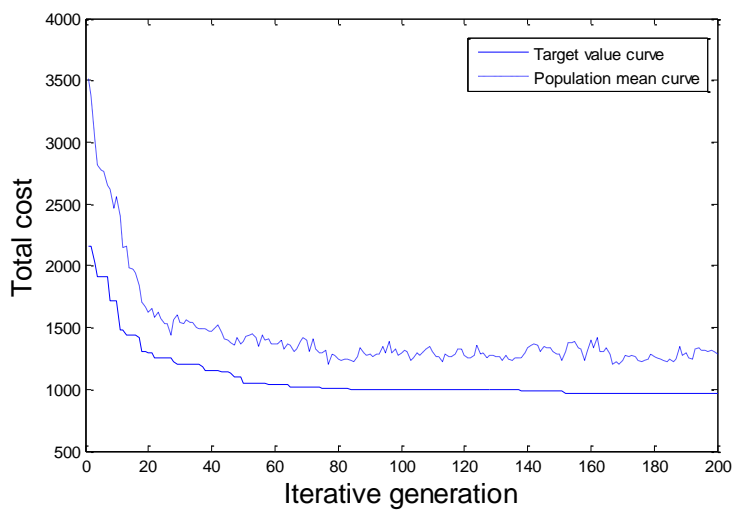

Figure 5. The minimum cost convergence graph of $10 \times 10$

Curve represents the optimal value evolution curve of each generation. With the constant evolution of the algorithm, the optimal objective function value tends to become more and more stable, indicating the correctness of the algorithm and convergence. The optimal processing sequence Gantt charts are shown in Fig. 6 and Fig. 7 (most likely processing time of triangular fu zzy numbers taken as proces sing time).

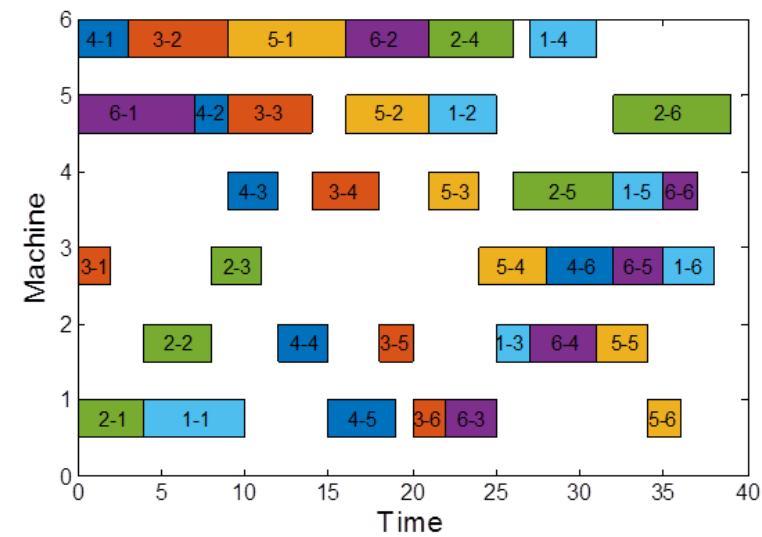

Figure 6. The optimal processing sequence Gantt chart of $6 \times 6$

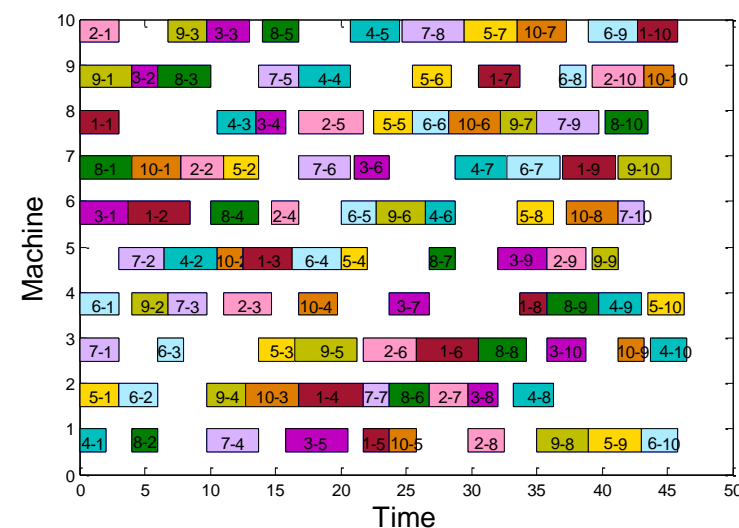

Figure 7. The optimal processing sequence Gantt chart of $10 \times 10$

\section{CONCLUSION}

In this paper, Job Shop problem with different due date windows was studied, considering the uncertainty of processing time, researchers use triangular fuzzy number to represent the processing time, a mathematical model was built to reduce the total cost of the production process and proposed an improved genetic algorithms to solve this problem. Finally, a numerical example was used to show the effectiveness of the algorithm. However, in real production systems, there are many uncertain factors, solving the more complex problems of uncertainty and how to achieve trade-offs between multiple objectives will be the future research directions.

\section{REFERENCES}

[1] Li Ping, Gu Xingsheng. Job Shop Scheduling with different due date windows under Uncertainty [J]. Management Science, 2004, 7(2): 22-26.

[2] Pistikopoulos E N. Uncertainty in process design and operations [J]. Computers \& Chemical Engineering, 1995, 19: 553-563.

[3] Yang Hongan, Wang zhoufeng. Interval number solving method for Job Shop Scheduling problem with processing time variability [J]. Computer Integrated Manufacturing Systems, 2014, 20(09): 2231-2240.

[4] Lei D. Population-based neighborhood search for job shop scheduling with interval processing time[J]. Computers \& Industrial Engineering, 2011, 61(4): 1200-1208.

[5] Azadeh A, Negahban A, Moghaddam M. A hybrid computer simulation-artificial neural network algorithm for optimization of dispatching rule selection in stochastic job shop scheduling problems [J]. International Journal of Production Research, 2012, 50(2): $551-566$.

[6] $\mathrm{Hu}$ Y, Yin M, Li X. A novel objective function for job-shop scheduling problem with fuzzy processing time and fuzzy due date using differential evolution algorithm [J]. The International Journal of Advanced Manufacturing Technology, 2011, 56(9-12): 11251138.

[7] Li Junqing, Pan Quanke. Solving Fuzzy Job-shop Scheduling Problems by a Hybrid Optimization Algorithm [J]. Journal of mechanical engineering, 2013, 49(23): 142-149.

[8] Yang Jianbin, Sun Shudong.Adaptive Genetic Algorithm for Fuzzy Job-shop Scheduling Problem [J]. Mechanical Science and Technlogy for Aerospace Engineering, 2013, 32(1): 16-21.

[9] Sakawa M, Kubota R. Fuzzy programming for multi objective job shop scheduling with fuzzy processing time and fuzzy due date through genetic algorithms $[\mathrm{J}]$. European Journal of operational research, 2000, 120(2): 393-407.

[10] Heilpern S. The expected value of a fuzzy number[J]. Fuzzy Sets and Systems, 1992, 47(1): 81-86. 
[11] Zhang Chaoyong, Rao Yunqing. An Improved Genetic Algorithm for the Job Shop Scheduling Problem [J]. Chinese Mechanical Engineering, 2005, 15(23): 2149-2153.
[12] Sakawa M, Mori T. An efficient genetic algorithm for job-shop scheduling problems with fuzzy processing time and fuzzy duedate[J]. Computers \& Industrial Engineering, 1999, 36(2): 325341 . 